\title{
Improving the Exact Security of Digital Signature Schemes
}

by

\author{
Leonid Reyzin \\ A.B., Computer Science (1996) \\ Harvard University
}

Submitted to the Department of Electrical Engineering and Computer Science in partial fulfillment of the requirements for the degree of

Master of Science in Computer Science

at the

\section{MASSACHUSETTS INSTITUTE OF TECHNOLOGY}

June 1999

(C) 1999 Massachusetts Institute of Technology

All rights reserved

Author

Department of Eleftrical Engineering and gmputer Science

May 21, 1999

Certified by

Silvio Micali

Professor of Computer Science and Engineering

Thesis Supervisor

Accepted by ..................

Arthur Smith

Chairman, Departmental Committee on Graduate Students
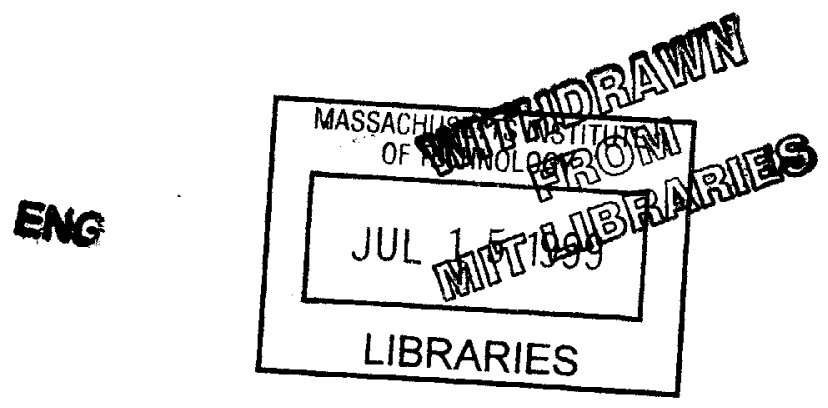


\title{
Improving the Exact Security of Digital Signature Schemes
}

by

\author{
Leonid Reyzin
}

Submitted to the Department of Electrical Engineering and Computer Science on May 21, 1999, in partial fulfillment of the

requirements for the degree of

Master of Science in Computer Science

\begin{abstract}
We provide two contributions to exact security analysis of digital signatures:

1. We put forward a new method of constructing Fiat-Shamir-like signature schemes that yields better "exact security" than the original Fiat-Shamir method; and

2. We extend exact security analysis to exact cost-security analysis by showing that digital signature schemes with "loose security" may be preferable for reasonable measures of cost.
\end{abstract}

Thesis Supervisor: Silvio Micali

Title: Professor of Computer Science and Engineering 


\section{Acknowledgments}

This thesis is largely based on the work I did jointly with my advisor, Silvio Micali. I greatly benefited from his many ideas, hours of fruitful discussions and well-timed encouragement.

Thanks to Salil Vadhan for pointing out an error in an earlier version of this work.

Thanks are also due to the many people who encouraged my interest in things mathematical. I am particularly grateful to my parents and first teachers, Marina and Natan Reyzin, my high school teachers Grigoriy Kondakov and Aleksey Belov-Kanel and my undergraduate advisor Michael O. Rabin.

To my wife, Monica Perez, thanks for all your patience and for the time you invested in this thesis. You helped me tremendously. And to our son, Jonathan, thanks for being such a good boy and letting me sleep at night. 


\section{Chapter 1}

\section{Introduction}

\subsection{Exact Security of Signature Schemes}

Goldwasser, Micali and Rivest's ([GMR88]) classical notion of security for a digital signature scheme is asymptotic in nature. They define a signature scheme to be secure if no polynomial-time adversary ("forger") can forge a signature after an adaptive chosenmessage attack. Because the current state of knowledge makes it impossible to prove the security of a signature scheme unconditionally (such a proof would, in particular, imply that $P \neq N P$ ), arguments that a signature scheme is secure are usually based on an assumption that some problem deemed hard (such as factoring or discrete logarithm) is impossible to solve in polynomial time. In essence, a proof of security amounts to a reduction from forging a signature to solving a computationally hard problem: if a polynomial-time forger exists, then we can use it to solve the hard problem in polynomial time.

It has been often pointed out that this asymptotic approach, which uses notions such as "polynomial time" and "sufficiently large," is too coarse for practical security recommendations. Knowing that no polynomial-time adversary has a better than exponentially small chance of forgery for a sufficiently large security parameter does not provide one with an answer to the practical problem of finding the appropriate security parameters to ensure security against adversaries with certain concrete capabilities.

Bellare and Rogaway ([BR96]) argue that, in order to be able to deduce concrete security recommendations, it is important to be precise in the reduction from a forger to the algorithm that solves the hard problem. For example, if one knows that factoring integers of length $l$ is no more than 100 times harder than breaking a certain signature scheme with security parameter $l$, then one could pick $l$ so that even $1 \%$ of the work required to factor integers of length $l$ is considered infeasible.

A reduction in which the difficulty of forging and the difficulty of solving the underlying hard problem are close is called tight; otherwise, it is called loose. (Naturally, "close," "tight" and "loose" are imprecise terms and make more sense when used in the comparative.) A scheme whose exact security is tightly related to the difficulty of factoring is also proposed in [BR96].

\subsection{Signature Schemes from ID Schemes}

A fruitful method for constructing signature schemes was introduced by Fiat and Shamir ([FS86]). Although claimed for a specific ID scheme based on modular square roots (a 
specific trapdoor function), the method works with a general commit-challenge-respond ID scheme. This is a three-pass, public-key-based, identification protocol, in which the prover sends a commitment to the verifier, receives a random challenge from the verifier, and then provides a response based on the commitment and the challenge. The verifier applies a verification procedure to the prover's public key, commitment, challenge and response, to verify that the prover really possesses the corresponding private key.

In order to change the ID scheme into a signature scheme, the verifier's random challenge is replaced by a publicly known "random" function $H$ evaluated at the prover's commitment and the message being signed. Thus, if the signer publishes the commitment and the response as the signature, anyone can convince oneself that the signer signed the message by first evaluating $H$ to find the random challenge and then applying the same verification procedure as in the ID scheme. If the verification procedure allows for recovery of the commitment from the challenge and the response (as it often does), then instead of publishing the commitment as part of the signature, the signer can publish the random challenge. Then the verifier needs to check that the value of $H$ evaluated at the signer's commitment and the message being signed is actually equal to the challenge given in the signature.

Intuitively, such a signature scheme is secure as long as the ID scheme is secure because the challenge produced by a random function is just as good as the challenge produced by a "live" verifier - an attacker cannot produce a challenge that suits its needs because $H$ is "random" (and hence, in particular, hard to invert).

Since [FS86], many such signature schemes have been proposed (see, e.g., [FFS88], [Bet88], [GQ88], [OO88], [MS88], [Sch89], [BDPW89], [Gir90], [BM90], [OS90], [Oka92], [Mic94], [Sho96], [Sch96], [PS96], [PS98], [OO98]). Many have been proven secure in the so called "random oracle" model (articulated in [BR93]). Essentially, these are proofs that rely on some cryptographic assumption (such as the hardness factoring or discrete logarithm) and the additional assumption that $H$ is a random oracle.

\subsection{The Contributions of this Paper}

This paper makes two contributions applicable to signature schemes in general.

1. The traditional way of turning ID schemes into signature schemes, as outlined above, results in signature schemes with loose reductions to the underlying hard problem. In alternative to this traditional method,

We propose a new method of constructing signature schemes from Fiat-Shamirlike ID schemes that results in schemes with tight reductions and better exact security.

Our method can be used on ID schemes based on trapdoor functions, and thus is less general than the "generalized" Fiat-Shamir (for example, it does not apply do discrete-log based ID schemes).

2. After proposing a method for creating signature schemes with tight reductions, we demonstrate that tightness of a reduction alone is insufficient if one wishes to maximize security while minimizing costs other than key length (e.g., signing time). It is indeed true that a tighter reduction allows for a lower security parameter, however: 
We show that a "loose" scheme can be so efficient that, though requiring a larger security parameter for a specified level of security, may deliver better performance (e.g., in signing time) than a "tight" scheme for the same level of security.

We submit that designers of digital signatures should be aware that "loose" signature schemes may provide more security for the same cost (or, equivalently, the same security for less cost) than "tight" signature schemes with respect to one or more reasonable measures of cost.

Our contributions are better illustrated in the concrete. To this end, we utilize the signature scheme and corresponding ID scheme of [Mic94] as a running example, henceforth called the $E$ scheme. Our contributions are thus exemplified as follows:

$1^{\prime}$. We analyze the exact security of the E scheme and demonstrate that its rexuction to factoring is quite loose (and a tighter analysis doesn't appear possible). We then propose a modified scheme called "E-swap" for which we prove a very tight reduction to factoring. (E-swap's performance is actually comparable to that of schemes currently used in practice.)

$2^{\prime}$. We demonstrate that although E-swap has better exact security than the E scheme, which of the two schemes to pick depends on what the main factor in the cost is. If the efficiency of verifying is of main concern, then E-swap should be chosen. If, however, the efficiency of signing is the main concern, then the E scheme can deliver more security for less cost. In fact, in that case the $\mathrm{E}$ scheme can deliver more security for less cost than even the schemes of [BR96].

We highlight that our point is not sacrificing security for efficiency. Quite contrary, we leverage efficiency in order to achieve better security. We submit that measuring the cost of a signature scheme accurately is just as important as measuring security accurately, and demonstrate that schemes with worse exact security may actually achieve better security for the same cost. We hope that this may further the applicability of exact security analysis.

\subsection{Roadmap}

We begin by introducing formal definitions and dealing with other preliminaries in Chapter 2. We introduce the E signature scheme and analyze its security in Chapter 3. Our new method for constructing signature schemes is given in Chapter 4 . We then show in Chapter 5 how to apply exact security analysis to choosing a digital signature scheme so as to optimize a given cost for a given level of security. 


\section{Chapter 2}

\section{Definitions}

Notation. We will denote by $A^{?}$ a (probabilistic) oracle algorithm; $A^{O}$ denotes that algorithm endowed with the specific oracle $O$, where $O:\{0,1\}^{*} \rightarrow\{0,1\}$. Because oracles returning more than one bit per question, as well as multiple oracles, can all be easily simulated by a single oracle returning one bit per question, we will slightly abuse this notation and speak of, for example, $A^{G, H}$, for some $G:\{0,1\}^{*} \rightarrow\{0,1\}^{k}$ and $H:\{0,1\}^{*} \rightarrow$ $\{0,1\}^{l}$. Additionally, in some cases we will need to allow one probabilistic oracle algorithm $A^{?}$ access to another probabilistic oracle algorithm $B^{\text {? }}$ with the oracle $O$; we will denote it by $A^{B^{O}}$. Note that because $B^{?}$ is probabilistic, it is not, strictly speaking, an oracle; rather, this notation implies that, for each query, $B^{O}$ gives a single answer selected, with the appropriate probability, from the set of all possible answers. When convenient and clear, we will omit the superscript when speaking about $A^{?}$ or $A^{O}$.

We will denote by $x \leftarrow A$ the fact that the probabilistic algorithm $A$ output $x$.

Signature Schemes. Our definition of a signature scheme follows the one found in [GMR88] and refined in [BR93] to allow for random oracles. As is common, we decouple the notions of a signature scheme and of its security.

Definition $1 A$ signature scheme with an oracle is a triple of probabilistic oracle algorithms $\Pi=\left(G_{e n}\right.$ ? Sign $^{?}$, Ver $\left.^{?}\right)$. Gen is a key generation algorithm that, given the security parameter $k$, outputs a key pair $(p k, s k) .{ }^{1}$ Commonly, $p k$ is called a public key and sk a secret key ( $s k$ is sometimes also called a private key). Sign is a signing algorithm: given a message $M$ and a secret key sk it returns a signature $x$. Ver is a verification algorithm: given a public key $p k$, a message $M$ and a purported signature $x$, it outputs "accept" or "reject."

$A$ signature $x$ on a message $M$ is called valid with respect to a public key pk and an oracle $H$ if $\operatorname{Ver}^{H}(p k, M, x)=$ "accept." The only required relationship between the three algorithms is that the algorithm Sign output valid signatures: that is, for any oracle $H$ and message $M$, if $(p k, s k) \leftarrow G e n^{H}\left(1^{k}\right)$ and $x \leftarrow S i g n^{H}(s k, M)$, then $x$ is a valid signature on $M$ with respect to $p k$ and $H$.

Signature schemes with more than one security parameter can be defined similarly.

\footnotetext{
${ }^{1}$ Usually, we are interested in the running time of Gen as a function of $k$ rather than $\log k$. Therefore, technically, we need to think of Gen as being given $k$ in unary notation. This is denoted by $1^{k}$.
} 
Note that in the above definition, we do not specify what the oracle $H$ is or what it means for a signature scheme to be secure. This is discussed below. Our definition of security is a modified version of that in [BR96], which is based on [BR93] and [GMR88]. This definition concerns itself with exact, rather than asymptotic, security.

Intuitively, we want to capture the following in our definition of security: there is no algorithm (called "forger") that, for a random oracle $H$, is able to produce new valid signatures with reasonable probability in reasonable time without knowing $s k$. Moreover, we should assume that an attacker can coerce the signer into signing some number of messages of the attacker's choice - to carry out the so-called "adaptive chosen-message attack" [GMR88]. We model this by giving the forger oracle access to the oracle $H$ and to the algorithm $\operatorname{Sign}^{H}(s k, \cdot)$.

Definition $2 A$ forger $F^{?, ?}$ is a probabilistic two-oracle algorithm that is given a security parameter $k$ and a public key $p k$ as input. The first oracle of $F$ is called $a$ hashing oracle and the second oracle is called a signature oracle. Let $H$ be a hashing oracle, and let $(p k, s k)=$ $G e n^{H}\left(1^{k}\right)$ for some $k$. We say that the forger succeeds if $(M, x) \leftarrow F^{H . S_{i g n}{ }^{H}(s k, \cdot)}\left(1^{k}, p k\right)$ and $x$ is a valid signature on $M$ and $F$ did not query its signature oracle on $M$.

We say that a forger $\left(t, q_{s i g}, q_{\text {hash }}, \varepsilon, \delta\right)$-breaks the signature scheme if, for a security parameter $k$, the following holds:

- its running time (plus the size of its description) does not exceed $t(k)$

- the number of its queries to the signature oracle does not exceed $q_{s i g}(k)$

- the number of its queries to the hashing oracle does not exceed $q_{h a s h}(k)$

- with probability at least $\delta(k), G e n^{H}\left(1^{k}\right)$ generates such a key $(p k, s k)$ that the probability of the forger's success on input $\left(1^{k}, p k\right)$ is at least $\varepsilon(k)$ (here, the probability of the forger's success is taken over a random choice of the oracle $H$, the random tape of the forger, the random tape of the signer to whom the forger addresses the chosen-message queries, but not the choice of $p k$ )

Finally, we say that a signature scheme is $\left(t, q_{\text {sig }}, q_{\text {hash }}, \varepsilon, \delta\right)$-secure if no forger $\left(t, q_{\text {sig }}\right.$, $\left.q_{\text {hash }}, \varepsilon, \delta\right)$-breaks it.

(As an aside for the reader familiar with the definition of [BR96], we point out that if a scheme is $\left(t, q_{s i g}, q_{h a s h}, \varepsilon \delta\right)$-secure in the sense of the [BR96], then it is $\left(t, q_{\text {sig }}, q_{\text {hash }}, E, \delta\right)$ secure in the sense of the above definition. We simply separate the component of the probability that is due to the selection of the public key.)

Measuring Signature Scheme Security. Now that we have defined what it means for a signature scheme to be secure, how do we actually prove anything about security? We will relate the security of a signature scheme to the difficulty of some problem; in our case, the difficulty of factoring. Let $G e n\left(1^{l}\right)$ be an algorithm generating $l$-bit products of two primes.

Definition 3 We will say that an algorithm $A(t, \varepsilon, \delta)$-factors integers generated by Gen if, for a given parameter $l$,

- A's running time (plus the size of its description) does not exceed $t(l)$ 
- with probability at least $\delta(l), G e n\left(1^{l}\right)$ generates such an integer $n$ that $A$ has at least $\varepsilon(l)$ probability (taken over only the random choices of the algorithm, not the choice of $n$ ) of producing the correct factors of $n$ on input $n$

We will say that factoring integers generated by Gen is $(t, \varepsilon, \delta)$-secure if no such $A$ exists.

Given this definition of the difficulty of a problem, we can then explain the security of a signature scheme $\Pi$ in the following terms, as suggested by [BR96]: if some problem is $\left(t^{\prime}, \varepsilon^{\prime}, \delta^{\prime}\right)$-secure, then $\Pi$ scheme is $\left(t, q_{\text {sig }}, q_{\text {hash }}, \varepsilon, \delta\right)$-secure. If $t$ is not much smaller than $t^{\prime}$ and $\varepsilon, \delta$ are not much larger than $\varepsilon^{\prime}, \delta^{\prime}$, even for a reasonably large $q_{s i g}$ and $q_{\text {hash }}$, then the reduction proving the security is called tight. 


\section{Chapter 3}

\section{The E Scheme}

\subsection{Signature and Verification Algorithms}

We describe the following ID and signature scheme from [Mic94], with similarities to the Ong-Schnorr ([OS90]) and the Guillou-Quisquater ([GQ88]) schemes.

Number Theory. Let $k$ and $l$ be two security parameters. Let $p_{1} \equiv 3(\bmod 8)$ and $p_{2} \equiv 7(\bmod 8)$ be two primes of approximately equal size and $n=p_{1} p_{2}$ be an $l$-bit integer (such $n$ is called a Williams integer [Wil80]). To simplify further computations, we will assume not only that $n>2^{l-1}$, but also that $\left|Z_{n}^{*}\right|=n-p_{1}-p_{2}+1>2^{l-1}$, and that $p_{1}+p_{2}-1<2^{l / 2+1}$. Let $Q$ denote the set of non-zero quadratic residues modulo $n$. Note that $|Q|>2^{l-3}$. Note also that for $x \in Q$, exactly one of its four square roots is also in $Q$ (this follows from the fact that -1 is a non-square modulo $p_{1}$ and $p_{2}$ and the Chinese remainder theorem). Thus, squaring is a permutation over $Q$. From now on, when we speak of "the square root of $x$," we mean the single square root in $Q$; by $x^{2^{-k}}$ we will denote the single $y \in Q$ such that $x=y^{2^{k}}$. Also note that 2 is a non-square modulo $p_{1}$ and a square modulo $p_{2}$ (because $\left(\frac{2}{p}\right)=(-1)^{\left(p^{2}-1\right) / 8}$ ), so $1 \in Q$ and $-1,2,-2 \notin Q$. In general, for any $x \in Z_{n}^{*}$, exactly one of $x,-x, 2 x,-2 x$ is in $Q$.

Following [GMR88], define $F_{0}(x)=x^{2} \bmod n, F_{1}(x)=4 x^{2} \bmod n$, and, for an $m$-bit binary string $\sigma=b_{1} \ldots b_{m}$, define $F_{\sigma}: Q \rightarrow Q$ as $F_{\sigma}(x)=F_{b_{m}}\left(\ldots\left(F_{b_{2}}\left(F_{b_{1}}(x)\right)\right) \ldots\right)=$ $x^{2^{m}} 4^{\sigma} \bmod n$ (note that $4^{\sigma}$ is a slight abuse of notation, because $\sigma$ is a binary string, rather than an integer; what is really meant here is 4 raised to the power of the integer represented in binary by $\sigma$ ). Because squaring is a permutation over $Q$ and $4 \in Q, F_{\sigma}$ is a permutation over $Q$.

Note that $F_{\sigma}(x)$ cán be efficiently computed by anybody who knows $n$. Also, if one knows $p_{1}$ and $p_{2}$, one can efficiently compute $x=F_{\sigma}^{-1}(y)$ (as shown by Goldreich in [Gol86]) by computing $s=1 / 4^{2^{-|\sigma|}} \bmod n$ and then letting $x=y^{2^{-|\sigma|}} s^{\sigma} \bmod n$ (these calculations can be done modulo $p_{1}$ and $p_{2}$ separately, and the results combined using the Chinese remainder theorem). However, if one does not know $p_{1}$ and $p_{2}$, then $F_{\sigma}^{-1}$ is hard to compute as shown in the Lemma below.

Lemma 1 If one can compute, for a given $y \in Q$ and two different strings $\sigma$ and $\tau$ of equal length, $x_{1}=F_{\sigma}^{-1}(y)$ and $x_{2}=F_{\tau}^{-1}(y)$, then one can factor $n$.

Proof The proof is by induction on the length of the strings $\sigma$ and $\tau$. 
If $|\sigma|=|\tau|=1$, then assume, without loss of generality, that $\sigma=0$ and $\tau=1$. Then $F_{0}\left(x_{1}\right) \equiv F_{1}\left(x_{2}\right) \equiv y \bmod n$, i.e., $x_{1}^{2} \equiv 4 x_{2}^{2} \bmod n$, i.e., $n \mid\left(x_{1}-2 x_{2}\right)\left(x_{1}+2 x_{2}\right)$. Note that $x_{1}, x_{2} \in Q$ and $\pm 2 \notin Q$, so $\pm 2 x_{2} \notin Q$, so $x_{1} \not \equiv \pm 2 x_{2} \quad(\bmod n)$, so $n$ does not divide either $x_{1}-2 x_{2}$ or $x_{1}+2 x_{2}$. Thus, by computing the gcd of $x_{1}+2 x_{2}$ and $n$, we can get either $p_{1}$ or $p_{2}$.

For the inductive case, let $\sigma$ and $\tau$ be two strings of length $m+1$. Let $\sigma^{\prime}$ and $\tau^{\prime}$ be their $m$-bit prefixes, respectively. If $F_{\sigma^{\prime}}\left(x_{1}\right) \equiv F_{\tau^{\prime}}\left(x_{2}\right) \quad(\bmod n)$, we are done by the inductive hypothesis. Otherwise, the last bit of $\sigma$ must be different from the last bit of $\tau$, so, without loss of generality, assume the last bit of $\sigma$ is 0 and the last bit of $\tau$ is 1 . Then $F_{0}\left(F_{\sigma^{\prime}}\left(x_{1}\right) \equiv F_{1}\left(F_{\tau^{\prime}}\left(x_{2}\right) \quad(\bmod n)\right.\right.$, and the same proof as for the base case works here.

The ID SCheme. The above lemma naturally suggests the following ID scheme. A user has $n$ as the public key and $p_{1}, p_{2}$ as the secret key. To prove his identity (i.e., that he knows $p_{1}$ and $p_{2}$ ) to a verifier, he commits to random $X \in Q$ and sends it to the verifier. The verifier produces a random $k$-bit challenge $\sigma$ and sends it to the prover (the user). The prover responds with $z=F_{0 \sigma}^{-1}(X)$ (note that $\sigma$ here is prefixed with a single 0 bit, whose use will be explained shortly). The verifier checks that $X=F_{0 \sigma}(z)=F_{\sigma}\left(z^{2}\right)$ and that $z \neq 0$ $(\bmod n)$. Informally, the security of this protocol is based on the fact that if the prover is able to respond to two different challenges $\sigma$ and $\tau$, then, by Lemma 1 , he knows $p_{1}$ and $p_{2}$. The 0 bit in front of $\sigma$ is to save the verifier from having to check that the prover's response is in $Q$ (which is a hard problem in itself) - instead, she just squares the prover's response and thus puts it in $Q$.

We will say no more about the security of this ID scheme because we are not concerned with it in this paper. We will, however, point out an efficiency improvement for the prover. First, as part of key generation, the prover computes, using the Chinese remainder theorem, $s=1 / 4^{2^{-k-1}} \bmod n$. Then, when committing to a random $X \in Q$, the prover randomly selects an $x \in Z_{n}^{*}$ and sets $X=x^{2^{k+1}} \bmod n$ (note that $X$ gets selected with uniform distribution as long as $x$ is so selected). Now, to respond to a challenge $\sigma$, the prover simply computes $z=x s^{\sigma} \bmod n$.

The E SCheme. The standard way to change the above ID scheme into a signature scheme is to replace the verifier with a random function $H:\{0,1\}^{*} \rightarrow\{0,1\}^{k}$. The exact steps of the algorithms Gen, Sign and Ver follow.

\section{Key Generation}

1. Generate two random primes $p_{1}=3 \bmod 8$ and $p_{2}=7 \bmod 8$ and $n=p_{1} p_{2}$ so that $n<2^{l}, n-p_{1}-p_{2}+1>2^{l}+1$ and $p_{1}+p_{2}-1<2^{l / 2+1}$

2. Generate coefficient $c=p_{2}{ }^{-1} \bmod p_{1}$ for use in the Chinese remainder theorem

3. Compute $u_{i}=\left(\frac{p_{i}+1}{4}\right)^{k+1} \bmod \frac{p_{i}-1}{2}$ for $i=1,2$ (note that $u_{i}$ is such that raising a square to the power $u_{i}$ modulo $p_{i}$ will compute its $2^{k+1}$ root)

4. Compute $s_{i}=\left(\frac{p_{i}+1}{4}\right)^{u_{i}} \bmod p_{i}$ for $i=1,2$

5. Compute $v=\left(s_{1}-s_{2}\right) c \bmod p_{1}$ and $s=s_{2}+v p_{2}$ to get $s=1 / 4^{2^{-(k+1)}} \bmod n$

6 . Output $n$ as the public key and $(n, s)$ as the secret key

\section{Signing}


1. Generate $X$ by picking a random $x \in Z_{n}^{*}$ and computing $X=x^{2^{k+1}} \bmod n$ (note that this step can be done off-line, before the message is known)

2. Compute $\sigma=H(X, M)$, and $z=F_{0 \sigma}^{-1}(X)$ via $t=s^{\sigma} \bmod n$ (this can be done via $t_{i}=s_{i}^{\sigma} \bmod p_{i}$ for $\left.i=1,2, v=\left(t_{1}-t_{2}\right) c \bmod p_{1}, t=t_{2}+v p_{2}\right)$ and $z=x t \bmod n$

3. Output $(z, \sigma)$

\section{Verifying}

1. Verify that $z \not 10(\bmod n)$ and compute $X=F_{\sigma}\left(z^{2}\right)$ via $t_{1}=z^{2^{k+1}} \bmod n$, $t_{2}=2^{\sigma} \bmod n, X=t_{1} t_{2} \bmod n$

2. Verify if $\sigma=H(X, M)$

\subsection{Security of the E scheme}

We state the following two theorems that give two different views of the exact security of the E scheme and demonstrate the tradeoff between running time and success probability. Their proofs use known methods (see Pointcheval and Stern [PS96] and Ohta and Okamoto [OO98]). Our probability analysis is new, however, and results in slightly tighter reductions.

Theorem 1 If there exists a forger that $\left(t, q_{s i g}, q_{\text {hash }}, \varepsilon, \delta\right)$-breaks the $E$ scheme with security parameters $l$ and $k$, then there exists an algorithm that $\left(t^{\prime}, \varepsilon^{\prime}, \delta\right)$-factors integers generated by Gen for

$$
\begin{aligned}
t^{\prime} & =2 t+2\left(q_{\text {sig }}+1\right) T_{1}+T_{2} \\
\varepsilon^{\prime} & =\frac{\varepsilon^{2}(1-2 \gamma)}{q_{\text {hash }}+1}-2^{-k},
\end{aligned}
$$

where $T_{1}$ is the time required to perform an $E$ signature verification, $T_{2}$ is the time required to factor $n$ given the conditions of Lemma 1 (essentially, a gcd computation) and $\gamma=$ $q_{\text {sig }}\left(q_{\text {hash }}+1\right) 2^{-l+3}$ (note that $\gamma$ is close to 0 for a large enough $l$ ).

\section{Proof}

MAIN IDEA. Let $F$ be a forger that $\left(t, q_{s i g}, q_{h a s h}, \varepsilon, \delta\right)$-breaks $E$ signature scheme. We will construct a factoring algorithm $A$ that uses $F$ to produce $y, z \in Z_{n}^{*}$ and $\sigma \neq \tau \in\{0,1\}^{k}$ such that $F_{\sigma}\left(z^{2}\right)=F_{\tau}\left(y^{2}\right)$. This will allow $A$ to factor $n$ by using the method given in the proof of Lemma 1.

The main idea of this proof is given by the "forking lemma" of [PS96]. It is to allow $F$ to run once to produce one forgery-a signature $(z, \sigma)$ on a message $M$ such that $\sigma=H(X, M)$ where $X=F_{\sigma}\left(z^{2}\right)$. Note that $F$ had to ask a hashing-oracle query on $(X, M)$-otherwise its probability of success is at most $2^{-k}$. Then, run $F$ the second time, giving the same answers to all the oracle queries before the query $(X, M)$. For $(X, M)$ give a new answer $\tau$. Then, if $F$ again forges a signature $(y, \tau)$ using $X$ and $M$, we will have achieved our goal.

Assuming $n$ is such that $F$ has probability at least $\varepsilon$ of success, the probability that $A$ will factor $n$ using this approach is roughly $\varepsilon^{2} / q_{\text {hash }}$, because $F$ needs to succeed twice and we have no guarantee that $F$ will choose to use $(X, M)$ for its second forgery and not any of its other $q_{\text {hash }}$ oracle queries. 
Details. We will now provide the details of the construction. We first have to show precisely how $A$ interacts with $F$. We will assume that $F$ performs the necessary bookkeeping and does not ask the same hash query twice. ${ }^{1}$ Note that $F$ may ask the same signature query twice, because the answers will most likely be different. We will also modify $F$ so that it will not output a signature $(z, \sigma)$ on a message $M$ unless it knows that the signature is correct; in particular, that means that $F$ has to first ask, in a hash query, for the value of $H\left(F_{\sigma}\left(z^{2}\right), M\right)$. This may increase the number of hash queries by one and the running time of $F$ by the cost of one signature verification.

$A$ first comes up with a random tape for $F$, remembers it, and runs $F$ on that tape. $A$ maintains two tables: a signature query table and a hash query table.

In order to answer a signature query on a message $M_{j}^{\prime}, A$ comes up with a random $z_{j} \in Q$ and $\sigma_{j}^{\prime} \in\{0,1\}^{k}$, computes $X_{j}^{\prime}=F_{\sigma_{j}^{\prime}}\left(z_{j}^{2}\right)$, and checks its signature query table to see if a signature query on $M_{j}^{\prime}$ has already been asked and $X_{j}^{\prime}$ used in answering it. If so, $A$ changes $z_{j}$ and $\sigma_{j}^{\prime}$ to the $z$ and $\sigma$ that were used in answering that query. Then $A$ adds the entry $\left(j, z_{j}, \sigma_{j}^{\prime}, X_{j}^{\prime}, M_{j}^{\prime}\right)$ to its signature query table and outputs $\left(z_{j}, \sigma_{j}^{\prime}\right)$.

In order to answer the $i$-th hashing query $\left(X_{i}, M_{i}\right), A$ first checks its signature query table to see if there is an entry $\left(j, z_{j}, \sigma_{j}^{\prime}, X_{j}^{\prime}, M_{j}^{\prime}\right)$ such that $\left(X_{j}^{\prime}, M_{j}^{\prime}\right)=\left(X_{i}, M_{i}\right)$. If so, it just outputs $\sigma_{j}^{\prime}$. Otherwise, it picks a random $\sigma_{i} \in\{0,1\}^{k}$, records in its hash query table the quadruple $\left(i, X_{i}, M_{i}, \sigma_{i}\right)$ and outputs $\sigma_{i}$.

Assume now that $F$ outputs a signature $(z, \sigma)$ on a message $M$. Let $X=F_{\sigma}\left(z^{2}\right)$. Because we modified $F$ to first ask a hash query on $(X, M)$, we have that, for some $i$, $(X, M, \sigma)=\left(X_{i}, M_{i}, \sigma_{i}\right)$ in the hash query table (it can't come from the signature query table, because $F$ is not allowed to forge a signature on a message for which it asked a signature query). $A$ finds such an $i$ in its table and remembers it.

$A$ now runs $F$ on the same random tape as the first time, giving the exact same answers to all $F$ 's queries before the $i$-th hash query (it can do so because it has all the answers recorded in the tables). Note that this means that $F$ will be asking the same $i$-th hash query $\left(X_{i}, M_{i}\right)$ as the first time. As soon as $F$ asks the $i$-th hash query, however, $A$ stops giving the answers from the tables and comes up with new answers at random, in the same manner as the first time. Let $\tau_{j}$ be the answer given to the $j$-th hash query for $j \geq i$.

Assume now that $F$ again outputs a signature $(y, \tau)$ on message $M^{\prime}$, and let $X^{\prime}=F_{\tau}\left(y^{2}\right)$. We know that $F$ had to ask a hash query on $\left(M^{\prime}, X^{\prime}\right)$. If it was the $i$-th hash query, then $X^{\prime}=X=X_{i}$, so $F_{\sigma}\left(z^{2}\right)=F_{\tau}\left(y^{2}\right)$. Thus, as long as $\sigma \neq \tau, A$ can factor $n$ by essentially performing a gcd computation (described in the proof Lemma 1 ).

Probability Analysis. First, we need the following lemma.

Lemma 2 Let $a_{1}, a_{2}, \ldots, a_{\lambda}$ be real numbers. Let $A=\sum_{\mu=1}^{\lambda} a_{\mu}$. Let $S=\sum_{\mu=1}^{\lambda} a_{\mu}{ }^{2}$. Then $S \geq \frac{A^{2}}{\lambda}$.

Proof Let $a=A / \lambda$ and $b_{\mu}=a-a_{\mu}$. Note that $\sum_{\mu=1}^{\lambda} b_{\mu}=\lambda a-\sum_{\mu=1}^{\lambda} a_{\mu}=A-A=0$. Then

$$
\sum_{\mu=1}^{\lambda} a_{\mu}^{2}=\sum_{\mu=1}^{\lambda}\left(a-b_{\mu}\right)^{2}=\lambda a^{2}-2 a \sum_{\mu=1}^{\lambda} b_{\mu}+\sum_{\mu=1}^{\lambda} b_{\mu}^{2} \geq \lambda a^{2}=\frac{A^{2}}{\lambda} .
$$

${ }^{1}$ This may slightly increase the running time of $F$, but we will ignore costs of simple table look-up for the purposes of this analysis. 
For this analysis, we assume that $n$ is such that $F$ has probability at least $\varepsilon$ of success. By assumption, such $n$ is generated with probability at least $\delta$.

First, consider the probability that $A$ 's answers to $F$ 's queries are distributed as those of the true oracles that $F$ expects. This is the case unless, for some signature query $j$, the hash value of $\left(X_{j}^{\prime}, M_{j}^{\prime}\right)$ has already been defined through a previous answer to a hash query (call this " $A$ 's failure to pretend"). Because $z_{j}$ is picked at random from $Q, X_{j}^{\prime}=F_{\sigma_{j}^{\prime}}\left(z_{j}^{2}\right)$ is a random element of $Q$. The probability of its collision with a value from a hash query in the same execution of $F$ is at most $\left(q_{\text {hash }}+1\right) /|Q|$; thus, the probability (taken over only the random choices of $A$ ) of $A$ 's failure to pretend in either of the two runs of $F$ is at most $2 q_{\text {sig }}\left(q_{\text {hash }}+1\right) /|Q| \leq q_{\text {sig }}\left(q_{\text {hash }}+1\right) 2^{-l+4}$.

We will now calculate the probability of the event that $F$ outputs a valid forgery based on the same hash query both times, assuming that $A$ does not fail to pretend. Let $p_{j}$ be the probability that, in one run, $F$ produces a valid forgery based on hash query number j. Clearly,

$$
\varepsilon=\sum_{j=1}^{q_{h a s h}+1} p_{j}
$$

$\left(q_{\text {hash }}+1\right.$ because we added an extra hash query to $F$ ). Let $p_{j, s}$ (for a sufficiently long binary string $s$ of length $m$ ) be the probability that, in one run, $F$ produces a valid forgery based on hash query number $j$ given that the string $s$ was used to determine the random tape of $F$ and the responses to all the queries of $F$ until (and not including) the $j$-th hash query. We have that

$$
2^{m} p_{j}=\sum_{s \in\{0,1\}^{m}} p_{j, s}
$$

Given such a fixed string $s$, the probability that $F$ produces a valid forgery based on the hash query number $j$ in both runs is $p_{j, s}^{2}$ (because the first forgery is now independent of the second forgery). Thus, the probability $q_{j}$ that $F$ produces a valid forgery based on the hash query number $j$ in both runs is

$$
q_{j}=\sum_{s \in\{0,1\}^{m}} 2^{-m} p_{j, s}^{2}=2^{-m} \sum_{s \in\{0,1\}^{m}} p_{j, s}^{2} \geq \frac{2^{-m}\left(p_{j} 2^{m}\right)^{2}}{2^{m}}=p_{j}^{2}
$$

(by Lemma 2).

The probability that $F$ produces a valid forgery based on the same hash query in both runs (assuming $A$ does not fail to pretend) is now

$$
\sum_{j=1}^{q_{\text {hash }}+1} q_{j} \geq \sum_{j=1}^{q_{h a s h}+1} p_{j}^{2} \geq \frac{\varepsilon^{2}}{q_{h a s h}+1}
$$

(by Lemma 2). The probability that $A$ does not fail to pretend is at least $1-q_{s i g}\left(q_{\text {hash }}+\right.$ 1) $2^{-l+4}$, so the probability that $A$ will end up with $\sigma, \tau, z$ and $y$ such that $F_{\sigma}\left(z^{2}\right)=F_{\mp}\left(y^{2}\right)$ is at least

$$
\frac{\varepsilon^{2}\left(1-q_{s i g}\left(q_{\text {hash }}+1\right) 2^{-i+4}\right)}{q_{\text {hash }}+1} .
$$


Subtract from that the probability that $\sigma$ and $\tau$ are equal (they are chosen at random) to get the desired result.

Theorem 2 If there exists a forger that $\left(t, q_{\text {sig }}, q_{\text {hash }}, \varepsilon, \delta\right)$-breaks the $E$ scheme with security parameters $l$ and $k$, such that $\varepsilon>2^{-k+1}\left(q_{\text {hash }}+1\right) /\left(1-q_{\text {sig }}\left(q_{\text {hash }}+1\right) 2^{-l+3}\right)$, then there exists an algorithm that $\left(t^{\prime}, \varepsilon^{\prime}, \delta\right)$-factors integers generated by Gen for

$$
\begin{aligned}
t^{\prime} & =\frac{\left(2 q_{\text {hash }}+3\right)\left(t+q_{\text {sig }} T_{1}\right)}{\varepsilon(1-\gamma)-2^{-k+1}\left(q_{\text {hash }}+1\right)}+T_{2} \\
\varepsilon^{\prime} & =\frac{1}{2}\left(1-\frac{1}{e}\right)^{2}>0.199
\end{aligned}
$$

where $T_{1}, T_{2}$ and $\gamma$ are as in Theorem 1.

\section{Proof}

MAIN IDEA. The idea is to iterate the $A$ from Theorem 1 sufficiently many times to get a constant probability of success. More specifically, run $F$ about $1 / \varepsilon$ times the first time, to achieve a constant probability of a successful forgery, and about $2 q_{h a s h} / \varepsilon$ times the second time, to achieve a constant probability of a successful forgery that uses the pair $(X, M)$.

Detalls. $A$ here works very similar to the $A$ of Theorem 1 . We will make exact same assumptions on $F$ and modifications to it. For the first run, $A$ simply repeats the procedure if $F$ does not succeed, up to $(\varepsilon(1-\gamma))^{-1}$ times. Every repetition starts entirely anew-with a new random tape for $F$ and new random answers from $A$.

Assuming $F$ succeeds, $A$ moves on to the second run. The second run is repeated until $F$ succeeds in producing a forgery on the $i$-th query, up to

$$
\frac{2 q_{\text {hash }}+2}{\varepsilon(1-\gamma)-2^{-k}\left(2 q_{\text {hash }}+2\right)}
$$

times. Every repetition starts with the same random tape for $F$ and the same answers for queries up to the $i$-th query; the answers to the $i$-th query and beyond are generated anew at random.

Probability ANALysis. We need the following lemma for this analysis. It is a restatement in probabilistic terms of the "heavy row lemma," found, in various forms, in [OO98], [PS96] and [FFS88], among others.

Lemma 3 Let $E$ be an event with probability $\alpha$. Let $E_{1}, \ldots, E_{\lambda}$ be disjoint events such that $\sum_{\mu=1}^{\lambda} \operatorname{Pr}\left[E_{\mu}\right]=1$. Let $\xi, 1 \leq \xi \leq \lambda$ be a random variable with the following distribution: $\operatorname{Pr}[\xi=\mu]=\operatorname{Pr}\left[E_{\mu} \mid E\right]$. Then

$$
\operatorname{Pr}_{\xi}\left[\operatorname{Pr}\left[E \mid E_{\xi}\right]>\frac{\alpha}{2}\right] \geq \frac{1}{2}
$$

Informally, this lemma can be stated as follows. Think of $E$ as the event of an experiment being successful, and each $E_{\mu}$ as the event of the experiment having certain preconditions. The probability of the experiment's success (regardless of preconditions) is $\alpha$. The lemma says that if the experiment actually succeeded, then it is quite likely (probability at least $1 / 2$ ) its preconditions were such that, if repeated with the same preconditions, its probability 
of success is at least $\alpha / 2$. That is, if the experiment succeeded, probably its preconditions were not too bad.

Proof In two sentences, the proof is simply that the set of preconditions that contribute only $\frac{\alpha}{2}$ to the success of $E$ cannot together contribute more than a half of all the successes of $E$. Thus, the other half of successes has to come from preconditions which contribute more than $\frac{\alpha}{2}$ to the success of $E$.

More formally, suppose $\operatorname{Pr}\left[\operatorname{Pr}\left[E \mid E_{\xi}\right]>\frac{\alpha}{2}\right]<\frac{1}{2}$. Then $\operatorname{Pr}\left[\operatorname{Pr}\left[E \mid E_{\xi}\right] \leq \frac{\alpha}{2}\right]>\frac{1}{2}$. Let $S=\left\{\mu \mid \operatorname{Pr}\left[E\left\{E_{\mu}\right] \leq \frac{\alpha}{2}\right\}\right.$, and let $E_{S}=\bigvee_{\mu \in S} E_{\mu}$. Then $\operatorname{Pr}[\xi \in S]>\frac{1}{2}$. But also $\operatorname{Pr}[\xi \in S]=$ $\operatorname{Pr}\left[E_{S} \mid E\right]=\frac{\operatorname{Pr}\left[E_{S} \wedge E\right]}{\operatorname{Pr}[E]}=\frac{\sum_{\mu \in S} \operatorname{Pr}\left[E \wedge E_{\mu}\right]}{\alpha}=\frac{\sum_{\mu \in S} \operatorname{Pr}\left[E \mid E_{\mu}\right] \operatorname{Pr}\left[E_{\mu}\right]}{\alpha}<\frac{\sum_{\mu \in S}(\alpha / 2) \operatorname{Pr}\left[E_{\mu}\right]}{\alpha} \leq \frac{1}{2}$, which is a contradiction.

For this analysis, we assume that $n$ is such that $F$ has probability at least $\varepsilon$ of success. By assumption, such $n$ is generated with probability at least $\delta$.

For the purposes of this part of the analysis, we will replace $A$ with $A^{\prime}$ that operates similarly to $A$, except for the following. In the first part of the algorithm, it picks a query number $i\left(1 \leq i \leq q_{\text {hash }}+1\right)$ at random and a string $s$ at random for the random tape of $F$ and answers to all its queries, up to the $i$-th hash query. Starting from the $i$-th hash query, it answers queries at random (rather than from the string $s$ ). $A^{\prime}$ considers a run successful only if the forgery is based on the $i$-th hash query.

We define "failure to pretend" the same way as in the proof of Theorem 1 . The probability that $F$ succeeds in producing a forgery in a single run based on the $i$-th hash query if $A^{\prime}$ does not fail to pretend is $\varepsilon /\left(q_{\text {hash }}+1\right)$. The probability that $A^{\prime}$ does not fail to pretend is at least $1-\gamma$. Thus, the probability of a successful forgery in a single first run is at least $\alpha=\varepsilon(1-\gamma) /\left(q_{h a s h}+1\right)$. If we now repeat the first run $\alpha^{-1}$ times (as long as $\alpha>0$ ), the probability of a successful forgery is at least

$$
1-(1-\alpha)^{\alpha^{-1}} \geq 1-1 / e
$$

(where $e$ is the base of the natural logarithm).

Assume that $F$ did produce a successful forgery, based on hash query number $i$ and string $s$. Let $p_{i, s}$ be the probability that, if $i$ and $s$ are picked as above, $F$ produces a successful forgery based on hash query number $i$ and string $s$. Note that $p_{i, s}$ is exactly the probability of a successful forgery on a second run. Also note that, by Lemma $3, \operatorname{Pr}\left[p_{i, s} \geq \alpha / 2\right] \geq 1 / 2$.

Assume now that indeed $p_{i, s} \geq \alpha / 2$. Subtract from it the probability that $\sigma$ and $\tau$ are the same (in that case, just like in the proof of Theorem $1, A$ does not have enough information to factor). If we now repeat the second run of $F\left(\alpha / 2-2^{-k}\right)^{-1}$ times (as long as $\alpha / 2>2^{-k}$ ), the probability that $A^{\prime}$ will be able to factor $n$ is at least

$$
1-\left(1-\left(\alpha / 2-2^{-k}\right)\right)^{\left(\alpha / 2-2^{-k}\right)^{-1}} \geq 1-1 / e
$$

Multiplying together the probability of success in the first run, the probability that $p_{i, s} \geq 1 / 2$ and the probability of success in the second run, we get that the probability of success of $A^{\prime}$ is at least

$$
\frac{(1-1 / e)^{2}}{2} \geq 0.199
$$

Now replace $A^{\prime}$ with $A$ to obtain a slight decrease in running time (by a factor of about 1.5) without decreasing the probability of success. The difference is that, in the first part of 
the algorithm, $A$ does not try to guess the number $i$ of the query on which $F$ will succeed, but rather accepts $F$ 's success on any query. This increases the success probability of a single run by a factor of $\left(q_{h a s h}+1\right)$ and thus decreases the running time of the first part by the same factor. We only need to show that this does not affect the success probability of the second part of the algorithm.

The only difference for the second part of the algorithm is in how the query number $i$ and the string $s$ are determined. We will show that the distribution of the pairs $(i, s)$ is the same for $A$ as for $A^{\prime}$. Intuitively, this is so because nothing changes in the distribution of $(i, s)$ simply because $A^{\prime}$ throws away, at random, successful forgeries by $F$ when they don't agree with its choice of $i$. Indeed, in the case of $A^{\prime}$, for a given pair $(i, s)$ the probability that it will be used in the second part is

$\operatorname{Pr}\left[i\right.$ and $s$ are picked by $A^{\prime} \mid F$ forges on the query that is picked by $\left.A^{\prime}\right]=$ $\frac{\operatorname{Pr}[i \text { and } s \text { are picked and } F \text { forges on query } i]}{\operatorname{Pr}\left[F \text { forges on the query that is picked by } A^{\prime}\right]}=$ $\operatorname{Pr}[F$ forges on query $i$ and $s$ is picked $]$ $\overline{\left(q_{\text {hash }}+1\right) \operatorname{Pr}\left[F \text { forges on the query that is picked by } A^{\prime}\right]}=$ $\operatorname{Pr}[F$ forges on query $i$ and $s$ is picked by $A$ for random tape and answers up to query $i]$ $\operatorname{Pr}[F$ forges $]$

$\operatorname{Pr}[i, s$ come out of the first run of $A \mid F$ forges $]$,

which is exactly the probability that $(i, s)$ is picked in the case of $A$.

The following two statements follow directly from the theorems just proved once we fix the parameters to be high enough to avoid dealing with small terms.

Corollary 1 If factoring l-bit integers generated by Gen is $\left(t^{\prime}, \varepsilon^{\prime}, \delta\right)$-secure, then the $E$ signature scheme is $\left(t, q_{\text {sig }}, q_{\text {hash }}, \varepsilon, \delta\right)$-secure for

$$
\begin{aligned}
q_{s i g} & \leq 2^{l-5} /\left(q_{\text {hash }}+1\right) \\
t & =t^{\prime} / 2-\left(q_{\text {sig }}+1\right) T_{1}-T_{2} / 2 \\
\varepsilon & =\max \left(2 \sqrt{\left(q_{\text {hash }}+1\right) \varepsilon^{\prime}}, 2^{-k / 2+1} \sqrt{q_{\text {hash }}+1}\right)
\end{aligned}
$$

Proof The corollary is simply the contrapositive of Theorem 1 with some low-order terms removed.

Note that the value for $t$ follows directly by solving for $t$ the equation for $t^{\prime}$ in the statement of Theorem 1 . The value for $\varepsilon$ is computed as follows: solve for $\varepsilon$ the equation for $\varepsilon^{\prime}$ to get

$$
\varepsilon=\sqrt{\left(q_{\text {hash }}+1\right)\left(\varepsilon^{\prime}+2^{-k}\right) /(1-2 \gamma)} .
$$

Observe that we are allowed to increase this $\varepsilon$, as this will only weaken the result. Note that the condition on $q_{s i g}$ ensures that $1-2 \gamma \geq 1 / 2$, so setting $\varepsilon$ to $\sqrt{2\left(q_{\text {hash }}+1\right)\left(\varepsilon^{\prime}+2^{-k}\right)}$ will not decrease it. If $\varepsilon^{\prime} \geq 2^{-k}$, then $\varepsilon^{\prime}+2^{-k} \leq 2 \varepsilon^{\prime}$, so setting $\varepsilon$ to $\sqrt{4\left(q_{\text {hash }}+1\right) \varepsilon^{\prime}}$ will not decrease it. Otherwise, we set $\varepsilon$ to $\sqrt{2\left(q_{\text {hash }}+1\right)\left(2^{-k}+2^{-k}\right)}$.

Corollary 2 If factoring l-bit integers generated by Gen is $\left(t^{\prime}, 0.199, \delta\right)$-secure, then the $E$ 
signature scheme is $\left(t, q_{s i g}, q_{\text {hash }}, \varepsilon, \delta\right)$-secure for

$$
t=\frac{\left(t^{\prime}-T_{2}\right) \varepsilon}{4 q_{\text {hash }}+6}-q_{\text {sig }} T_{1}
$$

as long as

$$
\begin{aligned}
q_{s i g} & \leq 2^{l-5} /\left(q_{\text {hash }}+1\right) \\
\varepsilon & \geq 2^{-k+3}\left(q_{\text {hash }}+1\right)
\end{aligned}
$$

Proof Similarly to Corollary 1, this corollary is simply the contrapositive of Theorem 2 with some low-order terms removed.

Solving for $t$ the equation for $t^{\prime}$ of Theorem 2 , we obtain

$$
t=\frac{\left(t^{\prime}-T_{2}\right)\left(\varepsilon(1-\gamma)-2^{-k+1}\left(q_{\text {hash }}+1\right)\right)}{2 q_{\text {hash }}+3}-q_{s i g} T_{1} .
$$

Conditions on $q_{s i g}$ and $\varepsilon$ ensure that $\left(\varepsilon(1-\gamma)-2^{-k+1}\left(q_{\text {hash }}+1\right) \geq \varepsilon / 2\right.$. Observe that we are allowed to decrease $t$, as this will only weaken the result, so we are allowed to replace $\varepsilon(1-\gamma)-2^{-k+1}\left(q_{\text {hash }}+1\right)$ by $\varepsilon / 2$.

Note also that Theorem 2 requires that $\varepsilon \geq 2^{-k+1}\left(q_{\text {hash }}+1\right) /(1-\gamma)$, which is also assured here by the conditions on $q_{s i g}$ and $\varepsilon$. 


\section{Chapter 4}

\section{The Swap Method}

\subsection{Motivation}

As exemplified by the proof of Theorems 1 and 2 above, all known results for the security of Fiat-Shamir-like signature schemes involve losing a factor of $q_{\text {hash }}$ (in either time or success probability) in the reduction from a forger to an algorithm that breaks the underlying hard problem (see, for example, [FS86], [Sch96], [PS96], [Sho96], [OO98]). While no proof exists that the loss of this factor is necessary, the problem seems inherent in the way signature schemes are constructed from ID schemes, as explained below.

The security of an ID scheme usually relies on the fact that a prover would be unable to answer two different challenges for the same commitment without knowing the private key. Therefore, in the proof of security of the corresponding signature scheme, we need to use the forger to get two signatures on the same commitment, as we did in the proof of Theorems 1 and 2. The forger, however, has any of its $q_{\text {hash }}$ queries to pick for the commitment for the second signature-hence, our loss of the factor of $q_{\text {hash }}$. We want to point out that $q_{\text {hash }}$ is a significant factor, and its loss definitely makes a reduction quite loose. This is because a reasonable bound on the number of possible hash queries of committed adversaries is about $q_{\text {hash }}=2^{80}$ (see Section 4.4).

We therefore devise a new method of constructing signature schemes from ID schemes so that any one signature from the forger is enough to break the underlying hard problem.

\subsection{Method}

Recall that in Fiat-Shamir-like signature schemes, the signer comes up with the commitment and then uses $H$ applied to the commitment and the message to produce the challenge. We propose that instead the signer first come up with the challenge and then use $H$ applied to the challenge and the message to produce the commitment. In a way, we swap the challenge and the commitment.

This method applies whenever the signer can compute the response given only the challenge and the commitment. It does not apply when information used during the generation of the commitment is necessary to compute the response. For example, it does not apply to discrete-logarithm-based ID schemes (such as the Schnorr scheme [Sch89]) in which the prover needs to know the discrete logarithm of the commitment in order to provide the response.

Additionally, in order to use this method, one needs to get around the problem that 
the commitment is selected from some structured set (such as $Q$ in the case of $\mathrm{E}$ ), while $H$ returns a random binary string. This problem can usually be easily solved. The only case known to us when it seems to present a real obstacle is in the scheme of Ohta and Okamoto $([\mathrm{OO} 88])$ in the case when an exponent $L$ is used such that $\operatorname{gcd}\left(L,\left(p_{1}-1\right)\left(p_{2}-1\right)\right)>2$.

The key-generation algorithm and the private key may need to be modified slightly in order to provide the signer with the additional information needed to compute the response from a random commitment, rather than from a commitment that it generated. The verification algorithm remains vastly unchanged.

In the next section, we exemplify our proposed method and explain why it results a tighter security reduction.

\subsection{E-swap}

\subsubsection{Description}

The scheme depends on two security parameters: $k$ and $l$. Let $H:\{0,1\}^{*} \rightarrow\{0,1\}^{l-1}$ be a random function.

Key Generation The key generation is the same as in the E scheme, except for one additional step (step 6) and extra information in the private key:

1. Generate two random primes $p_{1}=3 \bmod 8$ and $p_{2}=7 \bmod 8$ and $n=p_{1} p_{2}$ so that $n<2^{l}, n-p_{1}-p_{2}+1>2^{l}+1$ and $p_{1}+p_{2}-1<2^{l / 2+1}$

2. Generate coefficient $c=p_{2}{ }^{-1} \bmod p_{1}$ for use in the Chinese remainder theorem

3. Compute $u_{i}=\left(\frac{p_{i}+1}{4}\right)^{k+1} \bmod \frac{p_{i}-1}{2}$ for $i=1,2$ (note that $u_{i}$ is such that raising a square to the power $u_{i}$ modulo $p_{i}$ will compute its $2^{k+1}$ root)

4. Compute $s_{i}=\left(\frac{p_{i}+1}{4}\right)^{u_{i}} \bmod p_{i}$ for $i=1,2$

5. Compute $v=\left(s_{1}-s_{2}\right) c \bmod p_{1}$ and $s=s_{2}+v p_{2}$ to get $s=1 / 4^{2^{-(k+1)}} \bmod n$

6. If $u_{i}$ is odd, make it even by setting $u_{i}=u_{i}+\frac{p_{i}-1}{2}$ for $i=1,2$ (note that now $u_{i}$ is such that raising a square or its negative to the power $u_{i}$ modulo $p_{i}$ will compute its $2^{k+1}$ root)

7. Output $n$ as the public key and $\left(n, s, u_{1}, u_{2}, p_{1}, p_{2}\right)$ as the secret key

\section{Signing}

1. Generate a random $\sigma$ and compute $t=s^{\sigma} \bmod n$ (note that this step can be done off-line, before the message is known).

2. Compute $X=H(\sigma, M)$. We will assume $X \in Z_{n}^{*}$ (i.e., $(X, n)=1$ ), because the probability of $X \notin Z_{n}^{*}$ is at most $2^{-l / 2+2}$. If the Jacobi symbol $\left(\frac{X}{n}\right)=-1$, set $X=2 X \bmod n$. Now either $X$ or $n-X$ is in $Q$. Compute $z=F_{0} \sigma^{-1}( \pm X)$ via $x_{i}=X^{u_{i}} \bmod p_{i}$ for $i=1,2, v=\left(x_{1}-x_{2}\right) c \bmod p_{1}, x=x_{2}+v p_{2}$ and $z=x t \bmod n$.

3. Output $(z, \sigma)$.

\section{Verifying}


1. Verify that $z \not \equiv 0(\bmod n)$ and compute $X=F_{\sigma}\left(z^{2}\right)$ via $t_{1}=z^{2^{k+1}} \bmod n$, $t_{2}=2^{\sigma} \bmod n, X=t_{1} t_{2} \bmod n$ (this step is the same as for the $\mathrm{E}$ scheme).

2. Let $X^{\prime}=H(\sigma, M)$. If $X \equiv \pm X^{\prime} \quad(\bmod n)$ or $X \equiv \pm 2 X^{\prime} \quad(\bmod n)$, accept the signature (this step differs slightly from the $\mathrm{E}$ scheme).

\subsubsection{Security of E-swap}

Theorem 3 If there exists a forger that $\left(t, q_{s i g}, q_{h a s h}, \varepsilon, \delta\right)$-breaks the E-swap scheme with security parameters $l$ and $k$, then there exists an algorithm that $\left(t^{\prime}, \varepsilon^{\prime}, \delta\right)$-factors integers generated by Gen for

$$
\begin{aligned}
t^{\prime} & =t+2\left(q_{s i g}+q_{\text {hash }}+1\right) T_{1}+T_{2} \\
\varepsilon^{\prime} & =\varepsilon(1-\gamma)-\left(q_{\text {hash }}+q_{s i g}+1\right) 2^{-l / 2+2}
\end{aligned}
$$

where $T_{1}$ is the time required to perform an E-swap signature verification, $T_{2}$ is the time required to factor $n$ given the conditions of Lemma 1 (essentially, a gcd computation) and $\gamma=q_{\text {sig }}\left(q_{\text {hash }}+1\right) 2^{-k}$ (note that $\gamma$ is close to 0 for a large enough $k$ ).

\section{Proof}

MAIN IDEA. Familiarity with the proof of Theorem 1 will be helpful for understanding this proof.

Let $F$ be a forger that $\left(t, q_{s i g}, q_{h a s h}, \varepsilon, \delta\right)$-breaks E-swap signature scheme. Similarly to the proof of Theorem 1, we will construct an algorithm that, after interacting with $F$, will produce $y, z \in Z_{n}^{*}$ and $\sigma \neq \tau \in\{0,1\}^{k}$ such that $F_{\sigma}\left(z^{2}\right)=F_{\tau}\left(y^{2}\right)$.

The main idea is to answer each hash query on $(\sigma, M)$ with an $X$ computed via $X=$ $F_{\tau}\left(y^{2}\right)$ for a random $y \in Z_{n}^{*}$ and arbitrary $\tau$ that is different from $\sigma$. Then if $F$ forges a signature $(z, \sigma)$ on $M$, we will have $F_{\sigma}\left(z^{2}\right)=F_{\tau}\left(y^{2}\right)$ and will be able to factor $n$.

DETAILs. Just like in the proof of Theorem 1, we make the assumption that $F$ does not ask the same hash query twice and modify $F$ to output only correct signatures-in particular, to ask a hash query on $(\sigma, M)$ before outputting a signature $(z, \sigma)$ on $M$.

$A$ here also maintains two tables, for signature query answers and for hash query answers. To answer the $j$-th signature query on a message $M_{j}^{\prime}, A$ picks a random $\tau_{j}^{\prime} \in\{0,1\}^{k}$ and checks its signature query table to see if a signature query on $M_{j}^{\prime}$ has already been asked and if $\tau_{j}^{\prime}$ was used in answering it. If so, it returns the answer from the table. If not, it picks a random $y_{j}^{\prime} \in Q$ and a random $\mu_{j}^{\prime} \in\{1,-1,2,-2\}$, computes $X_{j}^{\prime}=F_{\tau_{j}^{\prime}}\left(y_{j}^{\prime 2}\right) / \mu_{j}^{\prime} \bmod n$, and checks if $X_{j}^{\prime}<2^{l-1}$. If not, it restarts with new random $y_{j}^{\prime}$ and $\mu_{j}^{\prime}$. The expected number of trials is less than 2 , because $X_{j}^{\prime}$ is a random element in $Z_{n}^{*}$ (because $F_{\tau_{j}^{\prime}}\left(y_{j}^{\prime 2}\right)$ is a random element in $Q$ and $\mu_{j}^{\prime}$ is also random) and $\left|Z_{n}^{*}\right|<n<2^{l}$. Once $X_{j}^{\prime}$ satisfies the desired condition, $A$ outputs $\left(y_{j}^{\prime}, \tau_{j}^{\prime}\right)$ as the signature and records $\left(j, y_{j}^{\prime}, \tau_{j}^{\prime}, X_{j}^{\prime}, M_{j}^{\prime}\right)$ in its signature query table.

To answer the $i$-th hash query on $\left(\tau_{i}, M_{i}\right), A$ first checks if an entry $\left(j, y_{j}^{\prime}, \tau_{j}^{\prime}, X_{j}^{\prime}, M_{j}^{\prime}\right)$ such that $\tau_{i}=\tau_{j}^{\prime}$ and $M_{i}=M_{j}^{\prime}$ already exists in its signature table. If so, it returns $X_{j}^{\prime}$. Otherwise, it picks $\tau_{i} \neq \sigma_{i}$, computes $y_{i}, \mu_{i}$ and $X_{i}$ in the same way as when answering a signature query, outputs $X_{i}$ and adds $\left(i, y_{i}, \tau_{i}, \mu_{i} X_{i}, M_{i}\right)$ to its hash query table.

Once $F$ outputs a forgery $(z, \sigma)$ on a message $M, A$ computes $X=F_{\sigma}\left(z^{2}\right)$ and searches its hash query table such an $i$ that $X=\mu_{i} X_{i}$ and $M_{i}=M$ (such an $i$ has to exist for the 
same reasons as in the proof of Theorem 1). In that case, $F_{\sigma}\left(z^{2}\right)=X=\mu_{i} X_{i}=F_{\tau_{i}}\left(y_{i}^{2}\right)$, and $\sigma \neq \tau_{i}$ because of the way $\tau_{i}$ was picked, so $A$ can easily factor $n$ by the method given the proof of Lemma 1

Probability ANALYSIS. For this analysis, we assume that $n$ is such that $F$ has probability at least $\varepsilon$ of success. By assumption, such $n$ is generated with probability at least $\delta$.

Define $A$ 's "failure to pretend" as the event that for some signature query $j$, the hash value of $\tau_{j}^{\prime}, M_{j}^{\prime}$ has been already defined through a previous hash query. The probability of this event for a given signature query is a most $\left(q_{\text {hash }}+1\right) 2^{-k}$, because $\tau_{j}^{\prime}$ is picked at random from $\{0,1\}^{k}$. Thus, the probability of $A$ 's failure to pretend is at most $q_{s i g}\left(q_{\text {hash }}+1\right) 2^{-k}$.

If $A$ does not fail to pretend, then $A$ 's answers to the queries are distributed just like those of the real oracles, except that $X$ in the answers to hash and signature queries is distributed uniformly over $Z_{n}^{*} \cap\{0,1\}^{l-1}$, thus excluding the multiples of $p_{1}$ and $p_{2}$ that a real hash function could possibly hit. Since there are at most $p_{1}+p_{2}-1<2^{l / 2+1}$ such multiples in $Z_{n}$, this will reduce the forger's probability of success by at most $\frac{2^{l / 2+1}}{2^{l-1}}=2^{-l / 2+2}$ per query.

So the probability of successfully factoring $n$ is

$$
\varepsilon^{\prime} \geq \varepsilon\left(1-q_{s i g}\left(q_{\text {hash }}+1\right) 2^{-k}\right)-\left(q_{\text {hash }}+q_{s i g}+1\right) 2^{-l / 2+2} .
$$

Theorem 4 If there exists a forger that $\left(t, q_{s i g}, q_{h a s h}, \varepsilon, \delta\right)$-breaks the $E$-swap scheme with security parameters $l$ and $k$, such that $\varepsilon>\left(q_{\text {hash }}+q_{\text {sig }}+1\right) 2^{-l / 2+2} /\left(1-q_{s i g}\left(q_{\text {hash }}+1\right) 2^{-k}\right)$, then there exists an algorithm that $\left(t^{\prime}, \varepsilon^{\prime}, \delta\right)$-factors integers generated by Gen for

$$
\begin{aligned}
t^{\prime} & =\frac{t+2\left(q_{\text {sig }}+q_{\text {hash }}+1\right) T_{1}}{\varepsilon(1-\gamma)-\left(q_{\text {hash }}+q_{s i g}+1\right) 2^{-l / 2+2}}+T_{2} \\
\varepsilon^{\prime} & =\left(1-\frac{1}{e}\right)>0.632
\end{aligned}
$$

where $T_{1}$ and $T_{2}$ are as in Theorem 3.

Proof Let

$$
\alpha=\varepsilon(1-\gamma)-\left(q_{h a s h}+q_{s i g}+1\right) 2^{-l / 2+2} .
$$

By assumption, $\alpha>0$. So if we repeat the algorithm constructed in the proof of Theorem 3 up to $1 / \alpha$ times (except for the final gcd computation, which need only be done once), we will get the desired $\varepsilon^{\prime}$, similarly to the proof of Theorem 2 .

Similarly to the E scheme, we have the following two corollaries.

Corollary 3 If factoring l-bit integers generated by Gen is $\left(t^{\prime}, \varepsilon^{\prime}, \delta\right)$-secure, then E-swap signature scheme is $\left(t, q_{s i g}, q_{\text {hash }}, \varepsilon, \delta\right)$-secure, where

$$
\begin{aligned}
q_{s i g} & \leq \min \left(2^{k-2} /\left(q_{\text {hash }}+1\right), \varepsilon 2^{l / 2-4}-q_{\text {hash }}-1\right) \\
t & =t-2\left(q_{s i g}+q_{\text {hash }}+1\right) T_{1}-T_{2} \\
\varepsilon & =2 \varepsilon^{\prime} .
\end{aligned}
$$


Proof The condition on $q_{s i g}$ ensures that $\left.\varepsilon(1-\gamma)-\left(q_{\text {hash }}+q_{s i g}+1\right) 2^{-l+2}\right) \geq \varepsilon / 2$. The rest follows, similarly to the proof of Corollary 1 , from solving the equations of Theorem 3 for $t$ and $\varepsilon$.

Corollary 4 If factoring l-bit integers generated by Gen is $\left(t^{\prime}, 0.632, \delta\right)$-secure, then the E-swap signature scheme is $\left(t, q_{s i g}, q_{\text {hash }}, \varepsilon, \delta\right)$-secure for

$$
\begin{aligned}
q_{s i g} & \leq \min \left(2^{k-2} /\left(q_{\text {hash }}+1\right), \varepsilon 2^{l / 2-4}-q_{\text {hash }}-1\right) \\
t & =\frac{\left(t^{\prime}-T_{2}\right) \varepsilon}{2}-2\left(q_{s i g}+q_{\text {hash }}+1\right) T_{1} .
\end{aligned}
$$

Proof The condition on $q_{s i g}$ ensures that $\left.\varepsilon(1-\gamma)-\left(q_{\text {hash }}+q_{s i g}+1\right) 2^{-l+2}\right) \geq \varepsilon / 2$. The rest follows, similarly to the proof of Corollary 2 , from solving the equations of Theorem 4 for $t$ and $\varepsilon$.

\subsection{Parameter Choice}

The formulas in the Corollaries 1-4 are quite different. Nonetheless, it is immediately clear that $E$-swap loses no factor of $q_{h a s h}$, neither in time nor in probability. This is a big advantage for E-swap because $q_{\text {hash }}$ can be quite big.

A fuller comparison, provided in the next section, depends on the actual values of the parameters $q_{s i g}, q_{h a s h}, k$ and $l$. Let us deal here, however, with the preliminary problem of assigning reasonable values to these parameters.

We believe it reasonable to set $q_{s i g}=2^{30}$ and $q_{\text {hash }}=2^{80}-1$. This is so because signature queries have to be answered by the honest signer (who may not be willing or able to sign more than a billion messages), while hash queries can be computed by the adversary alone (who may be willing to invest extraordinary resources). Notice that we recommend a higher value for $q_{\text {hash }}$ than suggested in [BR96].

We recommend setting $k=130$ for the E scheme and $k=112$ for E-swap. For the $\mathrm{E}$ scheme, this is so because, from Corollary 1 , we see that $2^{-k / 2+1} \sqrt{q_{\text {hash }}+1}$ has to be small. Therefore, we need $2^{-k / 2+41}$ to be small, and by setting $k=130$ we make it less than $10^{-7}$. (Notice that, mutatis mutandis we are again more conservative than [BR96].) For E-swap, this is so because $2^{k-2}$ has to be at least $q_{s i g}\left(q_{\text {hash }}+1\right)=2^{110}$ from Corollaries 3 and 4 .

As for $l$, notice that both $E$ and $E$-swap are immediately broken if the adversary succeeds in factoring the $l$-bit modulus. Therefore, $l$ ought to be at least 1000 . Given the above choices for the other parameters, such a minimum value for $l$ is large enough to make all the constraints involving $l$ in Corollaries 1-4 satisfied (for any reasonable $\varepsilon$ in the case of Corollaries 3 and 4 ). Thus, the value of $l$ depends on the presumed security of factoring, as discussed in the next chapter. 


\section{Chapter 5}

\section{The Case for Exact Security-Cost Analysis}

\subsection{The Costs of Security}

The desired level of security is usually dictated by the specific application. It is after settling on the desired amount of security that choosing among the various secure schemes becomes crucial. Indeed, when choosing a signature scheme, the goal is to maintain the desired level of security at the lowest possible cost. In a sense, picking a signature scheme is similar to shopping for an insurance policy for the desired face value.

The costs of a signature scheme, however, are quite varied. They may include the sizes of keys and signatures, the efficiencies of generating keys, signing and verifying, the amounts of code required, and even "external" considerations-such as the availability of inexpensive implementations or off-the-shelf hardware components. In this paper, we focus on the efficiencies of signing and verifying. These are particularly important when signing or verifying is performed by a low-power device, such as a smart card, or when signing or verifying needs to be performed in bulk quantities, as on a secure server.

It is for these costs, then, that below we compare the $\mathrm{E}$ and E-swap schemes. We also provide a comparison of the E scheme with the PRab scheme from [BR96], arguably the most practical among those tightly related to factoring. (The reason for choosing PRab rather than its PSS variant is that the latter is tightly related to RSA, and thus potentially less secure than factoring.)

\subsection{Comparison of $\mathrm{E}$ and E-swap}

The efficiency of signature verification in $\mathrm{E}$ is about the same as in E-swap. The security of E-swap is generally higher than the security of $E$ for the same security parameters. Therefore, if the efficiency of verifying is the only significant component in the cost, E-swap will be able to provide the same amount of security for less cost than $\mathrm{E}$.

A more difficult case to analyze is the case when the efficiency of signing is of main concern. We will limit our analysis to the case when we are only concerned with the on-line part of signing. In both cases, this involves mainly a modular exponentiation. Therefore, a variety of sophisticated algebraic methods can be used here, but these methods apply equally to $\mathrm{E}$ and E-swap. We thus find it simpler to compare the two under "standard" implementations using the Chinese remainder theorem (CRT). The E scheme then involves 
two exponentiations of a fixed $l / 2$-bit base to a $k$-bit power, modulo two $l / 2$-bit primes. One modular multiplication of two $l / 2$-bit numbers takes about $l^{2} / 4$ steps; about $3 k$ such multiplications are required ( $1.5 k$ for each of the primes), so the total amount of time required for on-line signing in the $\mathrm{E}$ scheme is about $3 \mathrm{kl}^{2} / 4$. Similar analysis applies for E-swap, except that about $1.5 l$ multiplications (1.5l/2 for each of the two primes) are required, so the total amount of time required for on-line signing in E-swap is about $3 l^{3} / 8$, not counting the (relatively small) cost of computing the Jacobi symbol. (In sum, on-line signing is $l /(2 k)$ times faster for $\mathrm{E}$ than for E-swap if one used the same value of $l$ for both. ${ }^{1}$ )

Let us now see how the security of the two schemes compares assuming the on-line signing costs are the same. Let $l_{E}$ and $k_{E}$ be the security parameters for $\mathrm{E}$, and $l_{E S}$ and $k_{E S}$ be the security parameters for E-swap. The on-line signing costs for $\mathrm{E}$ and E-swap are the same if

$$
l_{E S}=\left(2 k_{E} l_{E}^{2}\right)^{1 / 3} \text {. }
$$

The best known factoring algorithms take time about

$$
T(l)=C \exp \left(\left(\frac{64}{9}\right)^{1 / 3} l^{1 / 3}(\ln l)^{2 / 3}\right)
$$

for some constant $C$ [LL93]. Therefore, we will assume that factoring $l$-bit integers generated by $G e n$ is $\left(C^{\prime} T(l), 0.199, \delta\right)$-secure for some $\delta$ and some constant $C^{\prime}$. This means that, for a large enough $\varepsilon$ and small enough $q_{\text {sig }}$, the E scheme is about

$$
\left(\frac{\left(C^{\prime} T\left(l_{E}\right)-T_{2}\left(l_{E}, k_{E}\right)\right) \varepsilon}{4 q_{h a s h}+6}-q_{s i g} T_{1}\left(l_{E}, k_{E}\right), q_{s i g}, q_{h a s h}, \varepsilon, \delta\right)-\text { secure }
$$

and the E-swap scheme is about

$$
\left(\frac{\left(C^{\prime} T\left(l_{E S}\right)-T_{2}\left(l_{E S}, k_{E S}\right) \varepsilon\right.}{2}-2\left(q_{s i g}+q_{h a s h}+1\right) T_{1}\left(l_{E S}, k_{E S}\right), q_{s i g}, q_{h a s h}, \varepsilon, \delta\right)-\text { secure }
$$

(by Corollaries 2 and 4).

Keeping the signing costs equal, let us now find out when the $\mathrm{E}$ scheme becomes more secure than E-swap, that is, when

$$
\begin{array}{r}
\frac{\left(C^{\prime} T\left(l_{E}\right)-T_{2}\left(l_{E}, k_{E}\right)\right) \varepsilon}{4 q_{h a s h}+6}-q_{s i g} T_{1}\left(l_{E}, k_{E}\right)> \\
\frac{\left(C^{\prime} T\left(l_{E S}\right)-T_{2}\left(l_{E S}, k_{E S}\right) \varepsilon\right.}{2}-2\left(q_{s i g}+q_{h a s h}+1\right) T_{1}\left(l_{E S}, k_{E S}\right)
\end{array}
$$

Given the discussion of Section 4.4 and Equation (5.1), we will now plug in $q_{s i g}=2^{30}$, $q_{\text {hash }}=2^{80}-1, k_{E}=130, k_{E S}=112$, and $l_{E S}=\left(2 k_{E} l_{E}^{2}\right)^{1 / 3}$ to the above inequality. These values allow us to omit the terms in $T_{1}$ and $T_{2}$, noting that they decrease the security of E-swap much more than they decrease the security of $E$. Thus, we are now interested in

\footnotetext{
${ }^{1}$ Moreover, an optimization available to $\mathrm{E}$ but not to E-swap is precomputing some powers of the fixed base; this requires additional memory, so we will assume it is not implemented for the purposes of this analysis.
} 
when

$$
\frac{C^{\prime} T\left(l_{E}\right)}{2^{82}+2}>\frac{C^{\prime} T\left(\left(260 l_{E}^{2}\right)^{1 / 3}\right)}{2}
$$

After taking the natural logarithm of both sides, we are left with the inequality

$$
\left(\frac{64}{9}\right)^{1 / 3} l_{E}^{1 / 3}\left(\ln l_{E}\right)^{2 / 3}-\ln \left(2^{81}+1\right)>\left(\frac{64}{9}\right)^{1 / 3}\left(260 l_{E}^{2}\right)^{1 / 9}\left(\frac{1}{3} \ln 260+\frac{2}{3} \ln l_{E}\right)^{2 / 3},
$$

which holds as long as long as $l_{E} \geq 6749$.

Thus, at $l_{E}=6749, l_{E S}=2280, k_{E}=130, k_{E S}=112, \mathrm{E}$ and E-swap provide about the security and the same performance for on-line signing.

In Sum. The signing algorithm of the E scheme is so fast that

Provable security and signing efficiency are the same when E uses 6749-bit moduli and E-swap 2280-bit moduli.

In both cases, the security is that of factoring a 2280-bit integer generated by Gen. (The E scheme may actually be even more secure, but we cannot prove it!)

It just so happens that this computed level of security is currently considered adequate for many applications. (Therefore, for these applications E-swap is preferable: E-swap has faster verification for the same level of security, as well as shorter keys and, therefore, shorter signatures.)

However, whenever the application calls for an higher level of security, and the dominant cost is that of signing, then it is the "loose" E scheme that becomes preferable. In fact, Inequality (5.2) implies that the security gap between $E$ and E-swap, given the same performance, increases exponentially. In essence, this is so because, in order to guarantee the same performance, $l_{E S}$ is a fixed function of $l_{E}$ : i.e., by Equation (5.1), $l_{E S}=O\left(l_{E}^{2 / 3}\right)$. Thus security for both $E$ and E-swap is measured by Inequality (5.2) in terms of $l_{E}$, and the dominant factor in the exponent of the adversary's running time is $l_{E}^{1 / 3}$ for the $E$ scheme and $l_{E}^{2 / 9}$ for E-swap.

\subsection{Comparison of the E Scheme with Bellare-Rogaway's PRab}

The security of PRab is tightly related to that of modular square roots, rather than factoring. A factor of 2 in probability is lost (as compared to E-swap) when one relates the security of PRab to that of factoring. PRab's performance for on-line signing is about the same as E-swap's (PRab requires a few more Jacobi symbol computations, but no separate modular multiplication). ${ }^{2} \mathrm{~A}$ vastly similar analysis leads to the following conclusion:

Provable security and signing efficiency are the same when E uses 6619-bit moduli, and PRab 2251-bit moduli.

Also here this is a "cross-over" point: the gap in security for the same performance increases exponentially in favor of the $E$ scheme. As we can see, this cross-over point is just slightly more in favor of $E$ than the cross-over point of $E$ and E-swap. This is because of the factor of 2 difference in the security of E-swap and PRab.

\footnotetext{
${ }^{2}$ Unlike E-swap, however, PRab has no off-line component in signing.
} 


\section{Bibliography}

[BDPW89] Mike V. D. Burmester, Yvo Desmedt, Fred Piper, and Michael Walker. A general zero-knowledge scheme. In Quisquater and Vandewalle [QV89], pages $122-133$.

[Bet88] Thomas Beth. Efficient zero-knowledge identification scheme for smart cards. In Christoph G. Günther, editor, Advances in Cryptology-EUROCRYPT 88, volume 330 of Lecture Notes in Computer Science, pages 77-84. Springer-Verlag, 25-27 May 1988.

[BM90] Ernest F. Brickell and Kevin S. McCurley. An interactive identification scheme based on discrete logarithms and factoring (extended abstract). In Damgård [Dam90], pages 63-71.

[BR93] Mihir Bellare and Phillip Rogaway. Random oracles are practical: A paradigm for designing efficient protocols. In Proceedings of the 1st ACM Conference on

Com-

puter and Communication Security, November 1993. Revised version appears in http://www-cse.ucsd.edu/users/mihir/papers/crypto-papers .html.

[BR96] Mihir Bellare and Phillip Rogaway. The exact security of digital signatures: How to sign with and Rabin. In Maurer [Mau96], pages 399-416. Revised version appears in http://www-cse.ucsd.edu/users/mihir/papers/crypto-papers.html.

[Dam90] I. B. Damgård, editor. Advances in Cryptology-EUROCRYPT 90, volume 473 of Lecture Notes in Computer Science. Springer-Verlag, 1991, 21-24 May 1990.

[FFS88] Uriel Feige, Amos Fiat, and Adi Shamir. Zero-knowledge proofs of identity. Journal of Cryptology, 1(2):77-94, 1988.

[FS86] Amos Fiat and Adi Shamir. How to prove yourself: Practical solutions to identification and signature problems. In Odlyzko [Odl86], pages 186-194.

[Gir90] Marc Girault. An identity-based identification scheme based on discrete logarithms modulo a composite number. In Damgård [Dam90], pages 481-486.

[GMR88] Shafi Goldwasser, Silvio Micali, and Ronald L. Rivest. A digital signature scheme secure against adaptive chosen-message attacks. SIAM Journal on Computing, 17(2):281-308, April 1988.

[Gol86] Oded Goldreich. Two remarks concerning the Goldwasser-Micali-Rivest signature scheme. In Odlyzko [Od186], pages 104-110. 
[Gol88] S. Goldwasser, editor. Advances in Cryptology-CRYPTO '88, volume 403 of Lecture Notes in Computer Science. Springer-Verlag, 1990, 21-25 August 1988.

[GQ88] Louis Claude Guillou and Jean-Jacques Quisquater. A "paradoxical" indentitybased signature scheme resulting from zero-knowledge. In Goldwasser [Gol88], pages $216-231$.

[LL93] A. Lenstra and H. Lenstra, editors. The development of the number field sieve, volume 1554 of Lecture notes in Mathematics. Springer-Verlag, 1993.

[Mau96] Ueli Maurer, editor. Advances in Cryptology-EUROCRYPT 96, volume 1070 of Lecture Notes in Computer Science. Springer-Verlag, 12-16 May 1996.

[Mic94] Silvio Micali. A secure and efficient digital signature algorithm. Technical Report MIT/LCS/TM-501, Massachusetts Institute of Technology, Cambridge, MA, March 1994.

[MS88] Silvio Micali and Adi Shamir. An improvement of the Fiat-Shamir identification and signature scheme. In Goldwasser [Gol88], pages 244-247.

[Odl86] A. M. Odlyzko, editor. Advances in Cryptology-CRYPTO '86, volume 263 of Lecture Notes in Computer Science. Springer-Verlag, 1987, 11-15 August 1986.

[Oka92] Tatsuaki Okamoto. Provably secure and practical identification schemes and corresponding signature schemes. In Ernest F. Brickell, editor, Advances in Cryptology-CRYPTO '92, volume 740 of Lecture Notes in Computer Science, pages 31-53. Springer-Verlag, 1993, 16-20 August 1992.

[OO88] Kazuo Ohta and Tatsuaki Okamoto. A modification of the Fiat-Shamir scheme. In Goldwasser [Gol88], pages 232-243.

[OO98] Kazuo Ohta and Tatsuaki Okamoto. On concrete security treatment of signatures derived from identification. In Hugo Krawczyk, editor, Advances in Cryptology-CRYPTO '98, volume 1462 of Lecture Notes in Computer Science, pages 354-369. Springer-Verlag, 23-27 August 1998.

[OS90] H. Ong and C. P. Schnorr. Fast signature generation with a Fiat Shamir-like scheme. In Damgård [Dam90], pages 432-440.

[PS96] David Pointcheval and Jacques Stern. Security proofs for signature schemes. In Maurer [Mau96], pages 387-398.

[PS98] Guillaume Poupard and Jacques Stern. Security analysis of a practical "on the fly" authentication and signature generation. In Kaisa Nyberg, editor, Advances in Cryptology-EUROCRYPT 98, volume 1403 of Lecture Notes in Computer Science, pages 422-436. Springer-Verlag, May 31-June 41998.

[QV89] J.-J. Quisquater and J. Vandewalle, editors. Advances in CryptologyEUROCRYPT 89, volume 434 of Lecture Notes in Computer Science. SpringerVerlag, 1990, 10-13 April 1989.

[Sch89] C. P. Schnorr. Efficient identification and signatures for smart cards. In Quisquater and Vandewalle [QV89], pages 688-689. 
[Sch96] C. P. Schnorr. Security of $2^{t}$-root identification and signatures. In Neal Koblitz, editor, Advances in Cryptology-CRYPTO'96, volume 1109 of Lecture Notes in Computer Science, pages 143-156. Springer-Verlag, 18-22 August 1996.

[Sho96] Victor Shoup. On the security of a practical identification scheme. In Maurer [Mau96], pages 344-353.

[Wil80] Hugh C. Williams. A modification of the RSA public-key encryption procedure. IEEE Transactions on Information Theory, IT-26(6):726-729, November 1980. 